\title{
An Improved Clutter Suppression Method for Weather Radars Using Multiple Pulse Repetition Time Technique
}

\author{
Yingjie Yu and Yong Li \\ School of Electronics and Information, Northwestern Polytechnical University, Xian, China \\ Correspondence should be addressed to Yingjie Yu; sandy_yyj@126.com
}

Received 7 December 2016; Revised 18 March 2017; Accepted 28 March 2017; Published 9 April 2017

Academic Editor: Hiroyuki Hashiguchi

Copyright ( 2017 Yingjie Yu and Yong Li. This is an open access article distributed under the Creative Commons Attribution License, which permits unrestricted use, distribution, and reproduction in any medium, provided the original work is properly cited.

\begin{abstract}
This paper describes the implementation of an improved clutter suppression method for the multiple pulse repetition time (PRT) technique based on simulated radar data. The suppression method is constructed using maximum likelihood methodology in time domain and is called parametric time domain method (PTDM). The procedure relies on the assumption that precipitation and clutter signal spectra follow a Gaussian functional form. The multiple interleaved pulse repetition frequencies (PRFs) that are used in this work are set to four PRFs $(952,833,667$, and $513 \mathrm{~Hz}$ ). Based on radar simulation, it is shown that the new method can provide accurate retrieval of Doppler velocity even in the case of strong clutter contamination. The obtained velocity is nearly unbiased for all the range of Nyquist velocity interval. Also, the performance of the method is illustrated on simulated radar data for plan position indicator (PPI) scan. Compared with staggered 2-PRT transmission schemes with PTDM, the proposed method presents better estimation accuracy under certain clutter situations.
\end{abstract}

\section{Introduction}

The velocity and range ambiguity [1] is still one of the major limitations for Doppler weather radar observation. Given a radar wavelength $\lambda$, the relationship between the maximum unambiguous range $r_{a}$ and maximum unambiguous velocity $v_{a}$ can be presented as $v_{a} r_{a}=c \lambda / 8$, where $c$ is the speed of light. With a uniform pulse repetition frequency (PRF) $f_{\mathrm{PRF}}$ observation, $v_{a}$ can be shown as $v_{a}=\lambda f_{\mathrm{PRF}} / 4$. That means large maximum unambiguous velocity $v_{a}$ can be obtained by increasing the PRF. Along with the increase of maximum unambiguous velocity $v_{a}$ comes a decrease in maximum range $r_{a}$, and vice versa.

Various techniques have been proposed to overcome this problem [2-5], like dual-pulse repetition time (PRT) and staggered PRT $[3,6]$. The dual-PRT and staggered dualPRT techniques utilize two PRTs in an $n$ pulses transmission sequence. The dual-PRT method transmits two blocks of uniform PRT pulses with each block at a different PRT $\left(T_{1}, T_{1}, \ldots, T_{1}, T_{2}, T_{2}, \ldots, T_{2}\right)$. The optimal velocity estimates can be recovered from the joint analysis of the two Doppler measurements. This method is suitable for the low antenna rotation rate, because the radial velocity remains the same during the $n$ pulse intervals. The staggered PRT method is not restricted by this condition. The PRT alternates between two values $\left(T_{1}, T_{2}, T_{1}, \ldots, T_{1}, T_{2}\right)$. The interval of the maximum unambiguous velocity $v_{a}$ was well extended and successively adopted for the Next Generation Weather Radar (NEXRAD) network [7]. In order to improve the dealiasing success rate and obtain larger Nyquist velocity, the multiple PRT ( $M$ PRT) technique which utilizes multiple interleaved PRTs was proposed. Tabary et al. [8] presented a triple-PRT scheme implemented on the C-band French Radar Network. This technique works well when spectral width is smaller than $3 \mathrm{~m} / \mathrm{s}$ and SNR is high. A simultaneous multiple pulse repetition frequency (SMPRT) code [9] was introduced and evaluated. This technique can provide enough information to produce a high-resolution measured spectrum for each range gate. Tahanout et al. [10] proposed an optimal 9-PRT scheme for the suitable shape reproduction of the power spectrum of the radar signal.

These techniques which used nonuniform time series make filtering of clutter from the radar signal more complicated. Standard clutter filters cannot be applied directly 
to the nonuniform sampling scheme. Banjanin and Zrnic [11] developed a scheme which consists of two filters that operate sequentially; the overall filter is time-varying with periodically changing coefficients. Cho and Chornoboy [12] introduced a finite impulse response time-varying filter that is applied to $M$-PRT transmission. Siggia and Passarelli [13] proposed a Gaussian model adaptive processing (GMAP) algorithm that filters clutter and recovers weather spectrum after notching the spectrum around zero Doppler. Nguyen et al. [14] and Moisseev et al. [15] presented a parametric time domain method (PTDM) for staggered PRT observations, which can accurately estimate the spectral moments of precipitation echoes even with strong clutter.

In this work, we extend PTDM to $M$-PRT observations. This paper is organized as follows. In Section 2, staggered 2-PRT and $M$-PRT observation techniques are described. The PTDM for clutter suppression and spectral moments estimation is then presented in Section 3. Based on radar data simulations, Section 4 focuses on performance analysis of the proposed method. The error analyses of the proposed method and the staggered 2-PRT technique with PTDM for different noise and clutter scenarios are presented. In order to test the performance of the proposed method, different sample schemes techniques are used for plan position indicator (PPI) observations for comparison. In Section 5, discussions and conclusions are provided.

\section{M-PRT Techniques}

2.1. Uniform PRT. Assuming that $T$ is the PRT of the traditional uniform PRT technique, the autocorrelation calculated from return signal time series at lag $T$ is $\widehat{R}$. The maximum unambiguous velocity is $v_{a}=\lambda / 4 T$. The estimated velocity $v$ can be deduced from $\widehat{R}$ [1] as

$$
v=-\left(\frac{\lambda}{4 \pi T}\right) \arg (\widehat{R}),
$$

where $\arg ()$ is argument function.

2.2. Staggered 2-PRT. The staggered PRT method applies a pulse transmission sequence that changes intervals between $T_{1}$ and $T_{2}$. For $T_{2}>T_{1}$, the maximum unambiguous velocity $v_{a}$ will be extended as

$$
v_{a}=\frac{\lambda}{4\left(T_{2}-T_{1}\right)} .
$$

The autocorrelations calculated from return signal time series at lags $T_{1}$ and $T_{2}$ are $\widehat{R}_{1}$ and $\widehat{R}_{2}$. The Doppler velocities $v_{1}$ and $v_{2}$ can be deduced from $\widehat{R}_{1}$ and $\widehat{R}_{2}$, respectively, with (1).

Two approaches have been introduced to deal with the velocity aliasing. One algorithm uses the ratio of autocorrelations [7], given by

$$
v=\frac{\lambda}{4\left(T_{2}-T_{1}\right)} \arg \left(\frac{\widehat{R}_{1}}{\widehat{R}_{2}}\right) .
$$

If the argument of $\widehat{R}_{1} / \widehat{R}_{2}$ exceeds the range of $(-\pi, \pi)$, the estimated velocity will be ambiguous. The other technique utilizes the maximum unambiguous velocity to revise the aliasing velocity. Suppose $v_{a 1}$ and $v_{a 2}$ are the maximum unambiguous velocity for uniform PRTs $T_{1}$ and $T_{2}$. The dealiased velocity can be shown as

$$
\begin{gathered}
v=v_{1}+2 P v_{a 1}+\varepsilon_{1} \\
\text { or } v=v_{2}+2 Q v_{a 2}+\varepsilon_{2},
\end{gathered}
$$

where $P$ and $Q$ are the correct Nyquist interval number and $\varepsilon_{1}$ and $\varepsilon_{2}$ are estimate errors.

2.3. $M$-PRT. Mostly, $M$-PRT technique [9] can be regarded as an extension of the staggered 2-PRT technique. This technique chooses a transmission sequence which has several blocks, and every block is formed of $M$ different PRTs. Its transmitting scheme is $\left[T_{1}, T_{2}, \ldots, T_{M}, T_{1}, T_{2}, \ldots, T_{M}, \ldots\right]$. Similar to the staggered 2-PRT method, the maximum unambiguous velocity can be shown as

$$
v_{a}=\frac{\lambda}{4 \min \left(T_{i}-T_{j}\right)} ; \quad i \neq j .
$$

The relationship between the measured true velocity and the estimated velocities $v_{1}, v_{2}, \ldots, v_{M}$ is given by

$$
v^{(i)}=v_{i}+2 k_{i} v_{\mathrm{ai}}+\varepsilon_{i}, \quad i=1,2, \ldots, M .
$$

Finding the appropriate integers $\left(k_{1}, k_{2}, \ldots, k_{M}\right)$ which minimize the error $\varepsilon_{1}+\varepsilon_{2}+\cdots+\varepsilon_{M}$ is the way for the measured true velocity retrieval.

\section{Parametric Time Domain Method (PTDM)}

Bringi and Chandrasekar [16] have shown that the real and imaginary parts of radar signals follow zero mean normal distribution. Suppose $\mathbf{V}=\left[V_{1}, V_{2}, \ldots, V_{n}\right]^{t}$ is $n$ samples of received radar signals in one radar resolution volume with sample time $t_{1}, t_{2}, \ldots, t_{n}$. Hence, the complex vector can be expressed as the sum of two real Gaussian vectors representing in-phase and quadrature components, respectively, like

$$
V_{s}=I_{s}+j Q_{s} ; \quad s=1, \ldots, n .
$$

The multivariate density function of $\mathbf{V}$ [15] can be represented as

$$
\begin{aligned}
f(\mathbf{V}) & =\frac{1}{\pi^{n} \operatorname{det}(\mathbf{R})} \exp \left[-\left(\mathbf{V}^{*}\right)^{t} \mathbf{R}^{-1} \mathbf{V}\right] \\
& =\frac{1}{\pi^{n} \operatorname{det}(\mathbf{R})} \exp \left[-\operatorname{tr}\left(\mathbf{R}^{-1} \widehat{\mathbf{R}}_{V}\right)\right],
\end{aligned}
$$

where $\mathbf{R}=E\left[\mathbf{V}\left(\mathbf{V}^{*}\right)^{t}\right]$ is the covariance matrix and $\widehat{\mathbf{R}}_{V}=$ $\mathbf{V}\left(\mathbf{V}^{*}\right)^{t}$ is the sample covariance matrix. $\operatorname{tr}()$ is the trace function. $\operatorname{det}(\mathbf{R})$ is the determinant of $\mathbf{R}$.

Assuming that Doppler spectra of clutter and precipitation obey Gaussian distribution, the observed spectrum of 
Doppler velocity $v$ can be expressed as the summation of independent spectra coming from precipitation $S_{r}(v)$, clutter $S_{\mathrm{cl}}(v)$, and white noise $S_{\mathrm{N}}(v)$, as

$$
\begin{aligned}
S(v) & =S_{r}(v)+S_{\mathrm{cl}}(v)+S_{\mathrm{N}}(v) \\
S_{r}(v) & =\frac{P_{r}}{\sigma_{r} \sqrt{2 \pi}} \exp \left[-\frac{(v-\bar{v})^{2}}{2 \sigma_{r}^{2}}\right] \\
S_{\mathrm{cl}}(v) & =\frac{P_{\mathrm{cl}}}{\sigma_{\mathrm{cl}} \sqrt{2 \pi}} \exp \left[-\frac{v^{2}}{2 \sigma_{\mathrm{cl}}^{2}}\right] \\
S_{N}(v) & =\frac{2 \sigma_{\mathrm{N}}^{2} T_{\mathrm{s}}}{\lambda},
\end{aligned}
$$

where $P_{r}$ and $P_{\mathrm{cl}}$ are the precipitation signal and clutter power, $\sigma_{r}$ and $\sigma_{\mathrm{cl}}$ are the precipitation and clutter spectrum width, respectively, $\sigma_{\mathrm{N}}^{2}$ is the noise power, $\bar{v}$ is mean velocity, and $T_{\mathrm{s}}$ is sample time. Then, the covariance function can be obtained by fast Fourier transform (FFT) as

$$
\begin{aligned}
R(T, \theta)= & P_{r} \exp \left[-\frac{8 \pi^{2} \sigma_{r}^{2} T^{2}}{\lambda^{2}}\right] \exp \left[-j \frac{4 \pi \bar{v} T}{\lambda}\right] \\
& +P_{\mathrm{cl}} \exp \left[-\frac{8 \pi^{2} \sigma_{\mathrm{cl}}^{2} T^{2}}{\lambda^{2}}\right]+\sigma_{\mathrm{N}}^{2} \delta(T),
\end{aligned}
$$

where $\theta$ is the vector of unknown parameters, $\theta=\left[P_{r}, P_{\mathrm{cl}}, \sigma_{r}\right.$, $\left.\sigma_{\mathrm{cl}}, \bar{v}, \sigma_{\mathrm{N}}^{2}\right] . T$ is the temporal variable.

For $M$-PRT transmission, the element in the covariance matrix $\mathbf{R}$ can be written as

$$
\begin{array}{r}
\mathbf{R}(k, l) \\
=R\left[\operatorname{int}\left(\frac{l-k}{M}\right)\left(T_{1}+T_{2}+\cdots+T_{M}\right)+m a_{m}\right] ; \\
k, l=1, \ldots, n,
\end{array}
$$

where

$$
\begin{aligned}
m & =\bmod (l-k, M) \\
\mathbf{a} & =\left[a_{1}, a_{2}, \ldots, a_{M-1}\right] \\
& =\left[T_{1}, T_{1}+T_{2}, \ldots, T_{1}+T_{2}+\cdots+T_{M-1}\right] .
\end{aligned}
$$

Here, int is integral function. mod is the remainder of a division.

Using the multivariate density function (8), the loglikelihood estimation of $\theta$ is as follows:

$$
\ln L(\theta)=-\ln [\operatorname{det} \mathbf{R}(\theta)]-\operatorname{tr}\left[\mathbf{R}^{-1}(\theta) \widehat{\mathbf{R}}_{V}\right] .
$$

The parameter vector $\theta$ can be estimated by minimizing $\min [-\ln L(\theta)]$.

\section{Analysis of the Performance}

4.1. Simulation of Radar Signal. The main task of this work is to discuss the accuracy of estimated velocity under different
TABLE 1: Parameters of the simulated radar signal.

\begin{tabular}{lc}
\hline Parameter & Value \\
\hline Wavelength $(\mathrm{m})$ & 0.03 \\
Mean velocity $(\mathrm{m} / \mathrm{s})$ & $-v_{a},-0.9 v_{a}, \ldots, 0.9 v_{a}, v_{a}$ \\
CSR $(\mathrm{dB})$ & 20,40 \\
SNR $(\mathrm{dB})$ & 20,40 \\
Precipitation spectrum width $(\mathrm{m} / \mathrm{s})$ & 4 \\
Clutter spectrum width $(\mathrm{m} / \mathrm{s})$ & 0.25 \\
Noise power $(\mathrm{dB})$ & 20 \\
\hline
\end{tabular}

TABLE 2: Range of unknown parameters.

\begin{tabular}{lc}
\hline Parameter & Range \\
\hline Mean velocity $(\mathrm{m} / \mathrm{s})$ & $\left(-v_{a}, v_{a}\right)$ \\
Clutter power $(\mathrm{dB})$ & $\left(0, S_{\text {tot }}+10\right)$ \\
Precipitation power $(\mathrm{dB})$ & $\left(0, S_{\text {tot }}+10\right)$ \\
Precipitation spectrum width $(\mathrm{m} / \mathrm{s})$ & $(1,5)$ \\
Clutter spectrum width $(\mathrm{m} / \mathrm{s})$ & $(0.1,0.3)$ \\
Noise power $(\mathrm{dB})$ & $\left(\sigma_{N}^{\text {sys }}, \sigma_{N}^{\text {sys }}+10\right)$ \\
\hline
\end{tabular}

clutter conditions. So, different values of mean velocity, signal-to-noise ratio (SNR), and clutter-to-signal ratio (CSR) are chosen to generate the observed Doppler spectra. We select a periodic scheme with 4 different PRTs: 1050, 1200, 1500 , and $1950 \mu \mathrm{s}$. Their greatest common factor is $T_{u}=$ $150 \mu \mathrm{s}$. The ratio of PRTs is $7 / 8 / 10 / 13$. The corresponding PRFs can be written as 952, 833, 667, and $513 \mathrm{~Hz}$. The parameters of the simulated X-band radar signal are shown in Table 1.

4.2. PTDM Implementation. Using the log-likelihood function (13) to retrieve the precipitation and clutter spectral moments, the convergence of a local minimum will lead to wrong estimations in some cases. In order to reduce the estimate errors, we should make sure that the results of minimum (13) come from the convergence of global minimum. According to Moisseev et al. [15], this problem can be solved by selecting the appropriate seed value, especially for mean velocity seed value selection which has the biggest effect on the convergence of the optimization procedure. Similar to Moisseev et al. [15], the search range of unknown parameters is given in Table 2. $S_{\text {tot }}$ is the total signal power, and $\sigma_{\mathrm{N}}^{\text {sys }}$ represents the system noise floor. Using the Nelder-Mead simplex method $[17,18]$, the estimated values of unknown parameters are retrieved. Figure 1 shows a scatter diagram of estimated velocities for 4-PRT technique using PTDM, when $\mathrm{CSR}=20 \mathrm{~dB}$. It can be revealed that the estimated velocities are accurately retrieved using this method. The mean velocities almost coincide with true velocities. The standard deviations and biases are presented in Figure 2. The results show that the standard deviations are less than $0.5 \mathrm{~m} / \mathrm{s}$ and the biases are near zero without increase.

The noise and clutter environment is complex and variable in radar detection. The simulation can be carried out 


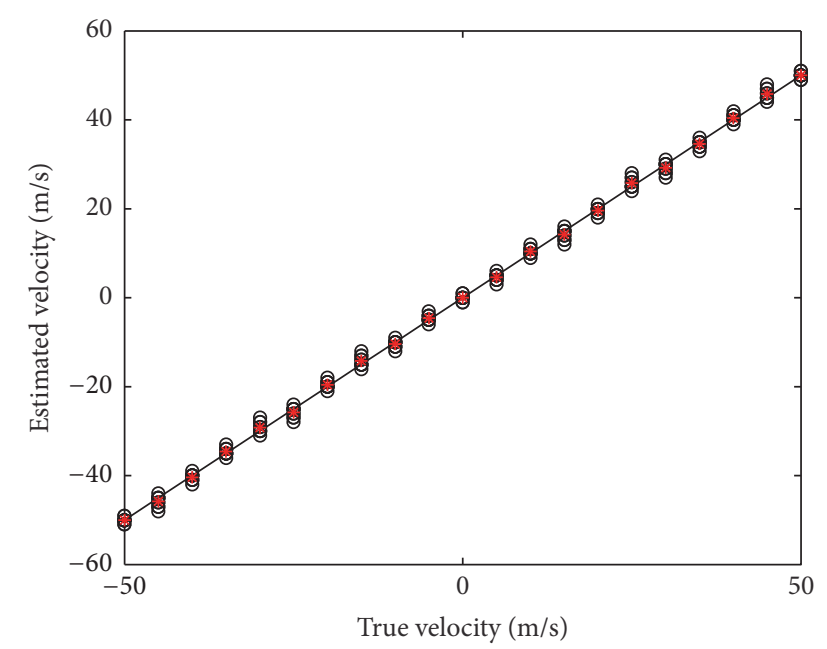

Figure 1: The true velocity (solid line) versus estimated velocities (hollow dot) from 30 simulations when $\mathrm{CSR}=20 \mathrm{~dB}$ and $\mathrm{SNR}=$ $40 \mathrm{~dB}$ using PTDM. The mean of estimated velocities of each input velocity is shown as a red asterisk character.

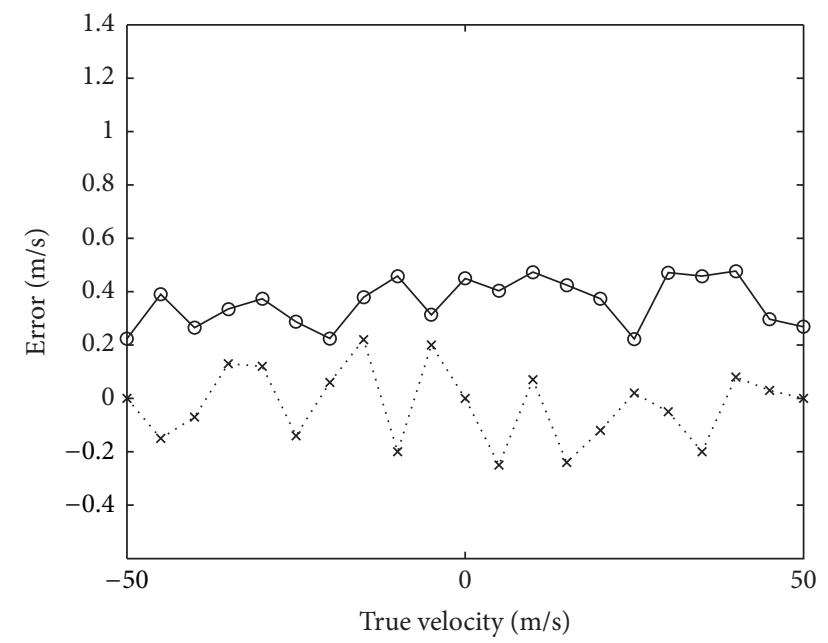

FIgURE 2: Standard deviation (solid line with hollow dot) and bias (dotted line with cross) of estimated velocities in Figure 1.

through two cases. The first one describes the conditions near the radar. The ground clutter echoes have various intensities. The other one considers the situations far away from the radar where ground clutter can be neglected. The simulated data are collected in the Nyquist velocity interval.

In the first type of simulation, the CSR changes from $-20 \mathrm{~dB}$ to $40 \mathrm{~dB}$. SNR is fixed to $+40 \mathrm{~dB}$ during the computation. Figure 3 shows the curves of standard deviation and bias of velocity estimates versus CSR, when applying 2PRT and $M$-PRT schemes. The clutter suppression method used in both transmission schemes is PTDM. The biases increase with CSR at a similar rate for the two PRT schemes. The absolute values of biases do not exceed $0.5 \mathrm{~m} / \mathrm{s}$. For the range from $-20 \mathrm{~dB}$ to $20 \mathrm{~dB}$, the standard deviation are smaller than $1 \mathrm{~m} / \mathrm{s}$ and obviously the 4-PRT schemes provide better estimation accuracy. The estimation error increases with CSR, especially from $20 \mathrm{~dB}$ to $30 \mathrm{~dB}$. When CSR > $30 \mathrm{~dB}$, the values tend to be almost steady near $1.8 \mathrm{~m} / \mathrm{s}$.

The second simulation is implemented for $0 \mathrm{~dB} \leq \mathrm{SNR} \leq$ $40 \mathrm{~dB}$ and $\mathrm{CSR}=-20 \mathrm{~dB}$. In Figure 4 , the biases and standard deviations are shown for the PTDM velocity estimates. In the $[15 \mathrm{~dB}, 40 \mathrm{~dB}]$ interval, the measured velocities for both PRT schemes are sufficiently accurate. Then, the bias and standard deviation increase with the decrease of SNR and reach the maximum near $\mathrm{SNR}=0 \mathrm{~dB}$. For the range from $5 \mathrm{~dB}$ to $40 \mathrm{~dB}$, the measured velocities are acceptable for the biases close to zero and the standard deviations are less than $0.6 \mathrm{~m} / \mathrm{s}$. The performance of 4-PRT schemes has better precision especially when the noise is strong (from $0 \mathrm{~dB}$ to $15 \mathrm{~dB})$.

In order to test the effectiveness of the proposed method, the $I$ and $Q$ data for plan position indicator (PPI) scan were simulated. Choosing a range-azimuth space of $30 \mathrm{~km} \times 60^{\circ}$, the resolution is $150 \mathrm{~m}$ and $1^{\circ}$. The radial velocity of each cell in the background is set to one constant. The fan-shape range from the center to $12 \mathrm{~km}$ is defined as ground clutter with zero Doppler velocity and spectral width $\sigma_{c}=0.25 \mathrm{~m} / \mathrm{s}$. At the range from $8.5 \mathrm{~km}$ to $25 \mathrm{~km}$, a circle region is set to simulate the wind shear in which the velocity field is unstable and changes rapidly in velocity magnitude and direction. The maximum velocity in wind shear field is $10 \mathrm{~m} / \mathrm{s}$ and spectral width is equal to $4 \mathrm{~m} / \mathrm{s}$. From Figure 4 , we have already known that the velocities estimates are desirable over a large range of SNR. This simulation is focused on comparing the observation results between different techniques under different CSR scenarios. SNR is fixed to $40 \mathrm{~dB}$. Figures $5(\mathrm{a})-5(\mathrm{c})$ correspond to a situation with $\mathrm{CSR}=10 \mathrm{~dB}$, Figures $5(\mathrm{~d})-5(\mathrm{f})$ to $\mathrm{CSR}=20 \mathrm{~dB}$, and Figures $5(\mathrm{~g})-5(\mathrm{i})$ to $\mathrm{CSR}=$ $30 \mathrm{~dB}$.

Figures 5(a), 5(d), and 5(g) illustrate the estimates of radial velocity sampled at single PRT $1050 \mu$ s using the pulse pair processing (PPP) method. The corresponding maximum unambiguous velocity is $7.14 \mathrm{~m} / \mathrm{s}$. The velocity aliasing can be seen obviously in the region of wind shear field. Because of the ground clutter, the estimation of velocity is highly influenced.

Figures 5(b), 5(e), 5(h), 5(c), 5(f), and 5(i) show the obtained velocity estimates for the 2-PRT (1050 and $1200 \mu s)$ and 4-PRT $(1050,1200,1500$, and $1950 \mu s)$ schemes with PTDM, respectively. The maximum unambiguous velocity of both transmission schemes is $50 \mathrm{~m} / \mathrm{s}$. The problem of velocity aliasing is well solved. Compared with Figures 5(b) and 5(e), the clutter is filtered out better in Figures 5(c) and 5(f). For the case $\mathrm{CSR}=30 \mathrm{~dB}$, the clutter filtering results are almost the same.

\section{Conclusions}

In this paper, a technique applying the parametric time domain method to the $M$-PRT transmission schemes is proposed. Based on the assumption that the Doppler spectra of clutter and precipitation follow Gaussian shape, simulation studies are carried out using a case of $M$-PRT schemes. 


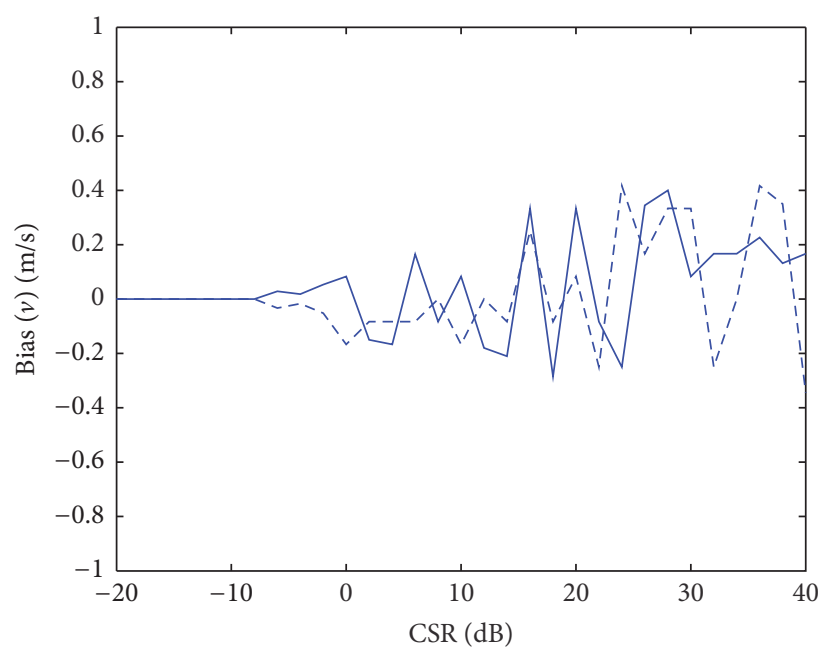

(a)

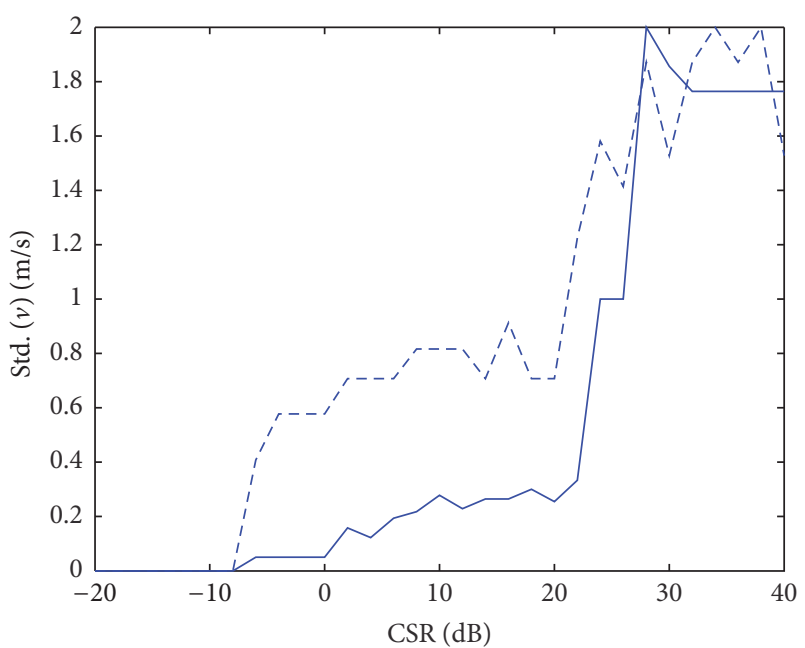

(b)

FIgURE 3: Bias (a) and standard deviation (b) of velocity estimates for the 4-PRT (solid line) and 2-PRT (dashed line) with SNR $=40 \mathrm{~dB}$ using the PTDM.

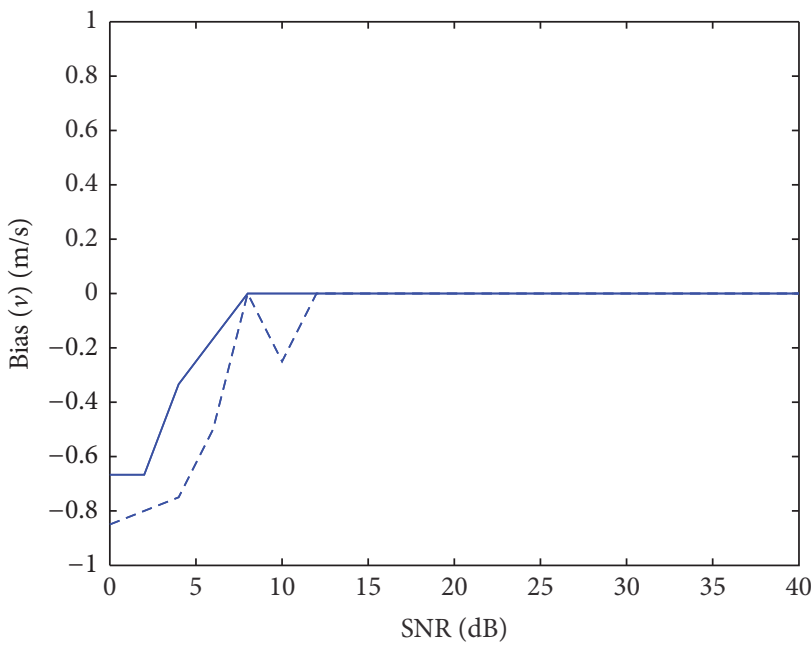

(a)

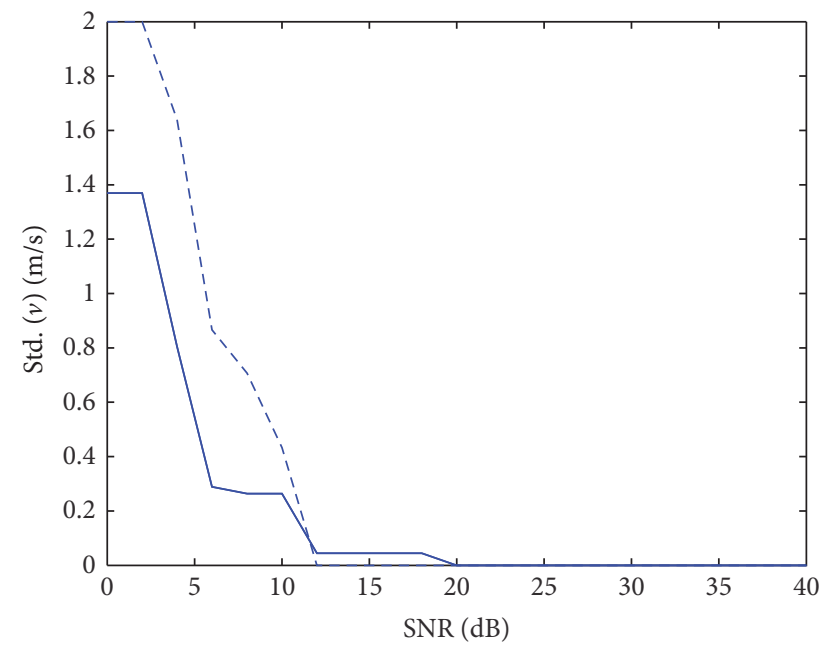

(b)

FIGURE 4: Bias (a) and standard deviation (b) of velocity estimates for the 4-PRT (solid line) and 2-PRT (dashed line) with CSR = -20 dB using the PTDM.

It is shown that the proposed method can effectively estimate Doppler velocity. Two cases are simulated to analyze the standard deviation and bias of measured velocities, which can help to compare the proposed method with 2-PRT transmission schemes with PTDM. The first one describes a situation where SNR is fixed to $40 \mathrm{~dB}$ and CSR varies. The biases are nearly the same for both of the methods. As long as CSR is lower than $20 \mathrm{~dB}$, the standard deviation of measured velocity using the proposed method are almost three times less than the 2-PRT transmission schemes with PTDM. The second type of simulation is performed for CSR $=-20 \mathrm{~dB}$ and $0 \mathrm{~dB} \leq \mathrm{SNR} \leq$ $40 \mathrm{~dB}$. The estimation error of measured velocities using the
4-PRT schemes is much better than that given by the 2-PRT schemes.

The PPI scan of measured velocity is built on simulated radar data with SNR $=40 \mathrm{~dB}$ and different CSRs. The results confirm that the proposed method not only solves Doppler velocity aliasing but also performs better in clutter suppression than 2-PRT schemes with PTDM especially when CSR $<$ $30 \mathrm{~dB}$.

The future works will focus on finding the optimal $M$ PRT schemes which minimize the estimation error and its applications to real air observation. The method to improve the computing efficiency is also the next emphasis of our research. 


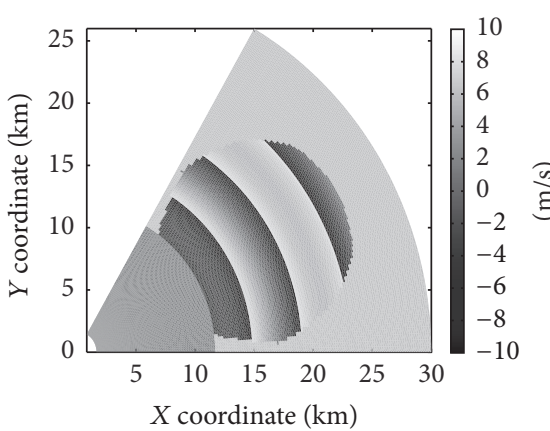

(a)

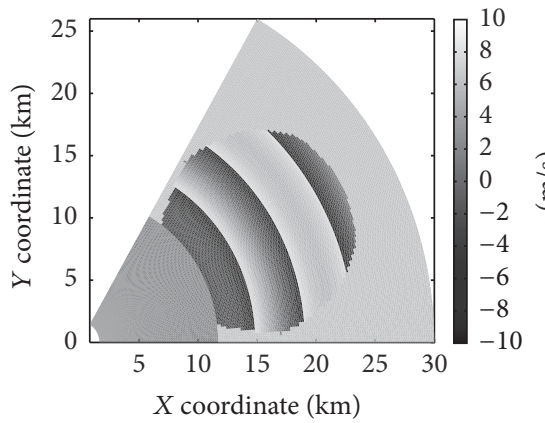

(d)

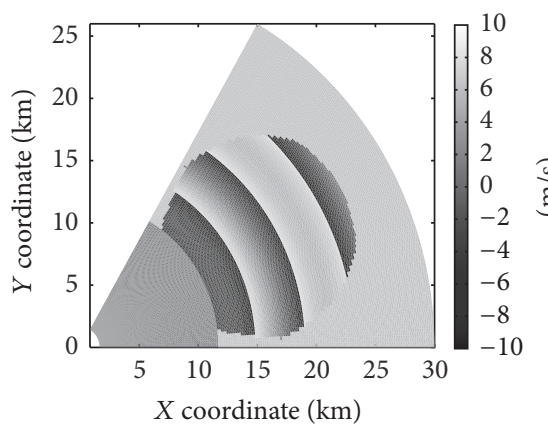

(g)

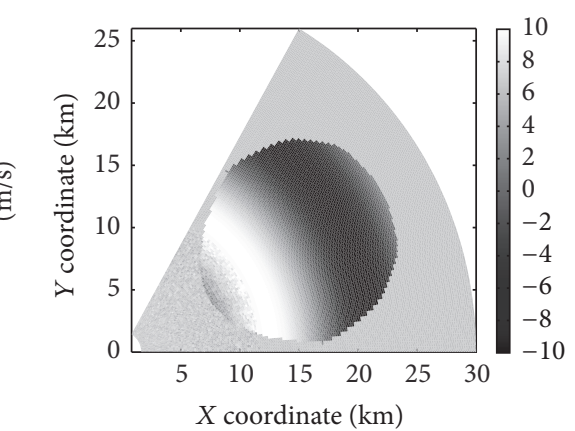

(b)

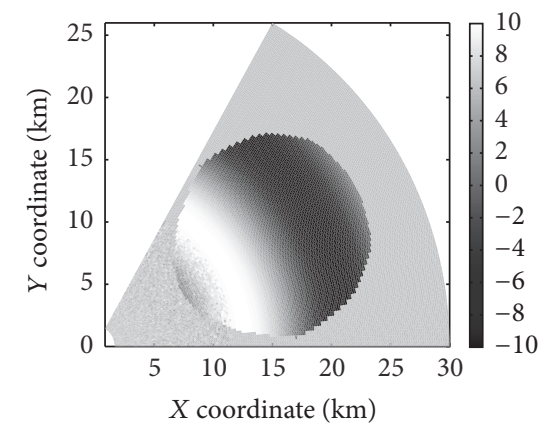

(e)

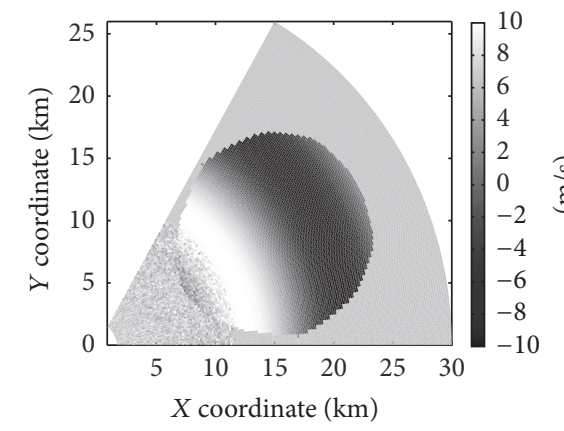

(h)

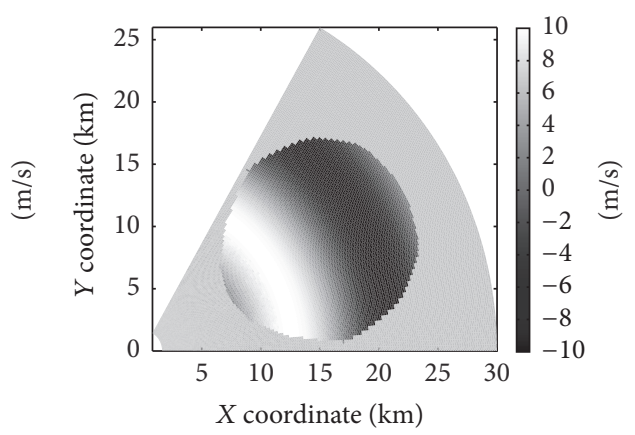

(c)

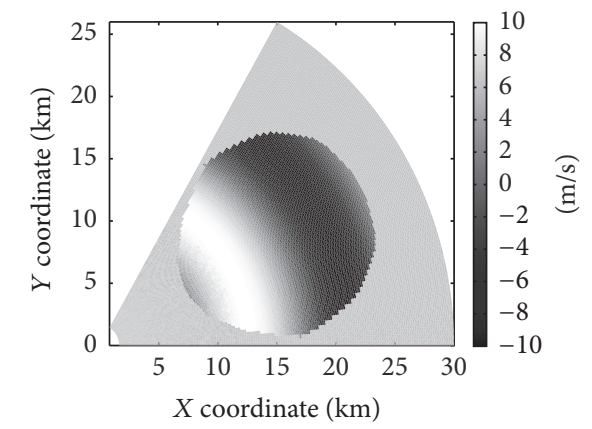

(f)

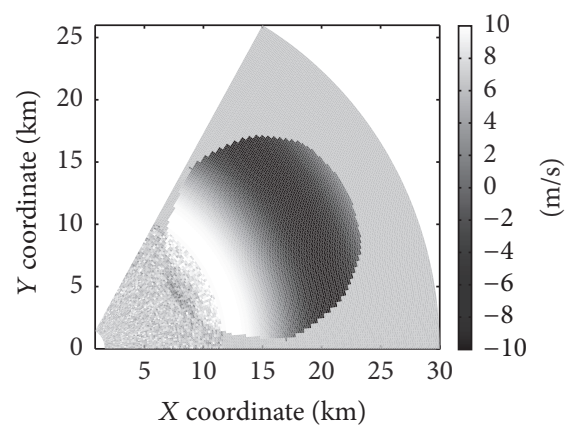

(i)

FIGURE 5: Radial velocity estimated from simulated $I$ and $Q$ radar data sampled at ((a), (d), (g)) unique PRT of $1050 \mu$ s, ((b), (e), (h)) 2-PRT $(1050$ and $1200 \mu \mathrm{s})$, and ((c), (f), (i)) 4-PRT (1050, 1200, 1500, and $1950 \mu \mathrm{s})$ with SNR $=40 \mathrm{~dB}$ and different CSR scenarios: (a) $-(\mathrm{c})$ CSR $=10 \mathrm{~dB}$, (d) $-(\mathrm{f}) \mathrm{CSR}=20 \mathrm{~dB}$, and (g)-(i) $\mathrm{CSR}=30 \mathrm{~dB}$. Note that the data are shown for $30 \mathrm{~km} \times 60^{\circ}$ space. The range and azimuth resolutions are $150 \mathrm{~m}$ and $1^{\circ}$, respectively. The corresponding unambiguous velocities are $7.14 \mathrm{~m} / \mathrm{s}$ for unique PRT and $50 \mathrm{~m} / \mathrm{s}$ for the other two schemes. The pulse pair processing (PPP) method is used in (a), (d), and (g). The PTDM is used in the others.

\section{Conflicts of Interest}

The authors declare that there are no conflicts of interest regarding the publication of this paper.

\section{Acknowledgments}

The authors wish to thank Dr. Wei Cheng for his continuous encouragement and support. The authors have benefited from in-house reports and information provided to them by the members of the Graduate Innovation Experimental Center.

\section{References}

[1] R. J. Doviak and D. S. Zrnic, Doppler Radar and Weather Observations, Academic Press, 1993.

[2] R. J. Doviak and D. Sirmans, "Doppler radar with polarization diversity," Journal of the Atmospheric Sciences, vol. 30, no. 4, pp. 737-738, 1973.

[3] D. S. Zrnic and P. Mahapatra, "Two methods of ambiguity resolution in pulse doppler weather radars," IEEE Transactions on Aerospace and Electronic Systems, vol. 21, no. 4, pp. 470-483, 1985.

[4] M. Sachidananda, D. S. Zrnic, and R. J. Doviak, "Signal design and processing techniques for WSR-88D ambiguity resolution. Part-1," Tech. Rep., National Severe Storms Laboratory, 1997. 
[5] M. Sachidananda, D. S. Zrnic, and R. J. Doviak, "Signal design and processing techniques for WSR-88D ambiguity resolution. Part-2," Tech. Rep., National Severe Storms Laboratory, 1998.

[6] Y. Golestani, V. Chandrasekar, and R. J. Keeler, "Dual polarized staggered PRT scheme for weather radars: analysis and applications," IEEE Transactions on Geoscience and Remote Sensing, vol. 33, no. 2, pp. 239-246, 1995.

[7] S. M. Torres, Y. F. Dubel, and D. S. Zrnić, "Design, implementation, and demonstration of a staggered PRT algorithm for the WSR-88D," Journal of Atmospheric and Oceanic Technology, vol. 21, no. 9, pp. 1389-1399, 2004.

[8] P. Tabary, F. Guibert, L. Perier, and J. Parent-du-Chatelet, "An operational triple-PRT Doppler scheme for the French radar network," Journal of Atmospheric and Oceanic Technology, vol. 23, no. 12, pp. 1645-1656, 2006.

[9] J. Pirttilä, M. S. Lehtinen, A. Huuskonen, and M. Markkanen, "A proposed solution to the range-Doppler dilemma of weather radar measurements by using the SMPRF codes, practical results, and a comparison with operational measurements," Journal of Applied Meteorology, vol. 44, no. 9, pp. 1375-1390, 2005.

[10] M. Tahanout, A. E. H. Adane, and J. Parent Du Chatelet, "An improved M-PRT technique for spectral analysis of weather radar observations," IEEE Transactions on Geoscience and Remote Sensing, vol. 10, pp. 1-11, 2015.

[11] Z. B. Banjanin and D. S. Zrnic, "Clutter rejection for doppler weather radars which use staggered pulses," IEEE Transactions on Geoscience and Remote Sensing, vol. 29, no. 4, pp. 610-620, 1991.

[12] J. Y. N. Cho and E. S. Chornoboy, "Multi-PRI signal processing for the terminal Doppler weather radar. Part I: clutter filtering," Journal of Atmospheric and Oceanic Technology, vol. 22, no. 5, pp. 575-582, 2005.

[13] A. D. Siggia and R. E. Passarelli, "Gaussian model adaptive processing (GMAP) for improved ground clutter cancellation and moment calculation," in Proceedings of the $3 r$ European Conference on Radar Meteorology (ERAD '04), pp. 67-73, Visby, Sweden, 2004.

[14] C. M. Nguyen, D. N. Moisseev, and V. Chandrasekar, "A time domain clutter filter for staggered PRT and dual-PRF measurements," in Proceedings of the IEEE International Geoscience and Remote Sensing Symposium (IGARSS '07), pp. 3325-3328, Barcelona, Spain, June 2007.

[15] D. N. Moisseev, C. M. Nguyen, and V. Chandrasekar, "Clutter suppression for staggered PRT waveforms," Journal of Atmospheric and Oceanic Technology, vol. 25, no. 12, pp. 2209-2218, 2008.

[16] V. N. Bringi and V. Chandrasekar, Polarimetric Doppler Weather Radar: Principles and Applications, Cambridge University Press, Cambridge, UK, 2001.

[17] J. A. Nelder and R. Mead, "A simplex method for function minimization," The Computer Journal, vol. 7, no. 4, pp. 308-313, 1965.

[18] J. C. Lagarias, J. A. Reeds, M. Wright, and P. E. Wright, "Convergence properties of the Nelder-Mead simplex method in low dimensions," SIAM Journal on Optimization, vol. 9, no. 1, pp. 112-147, 1999. 

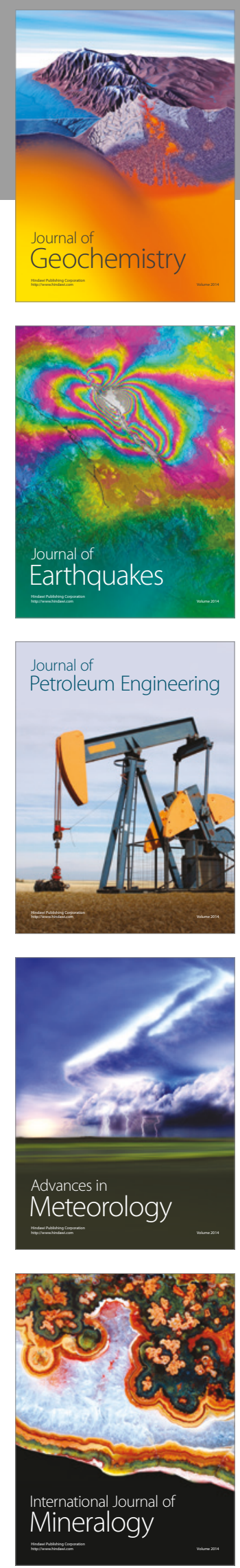
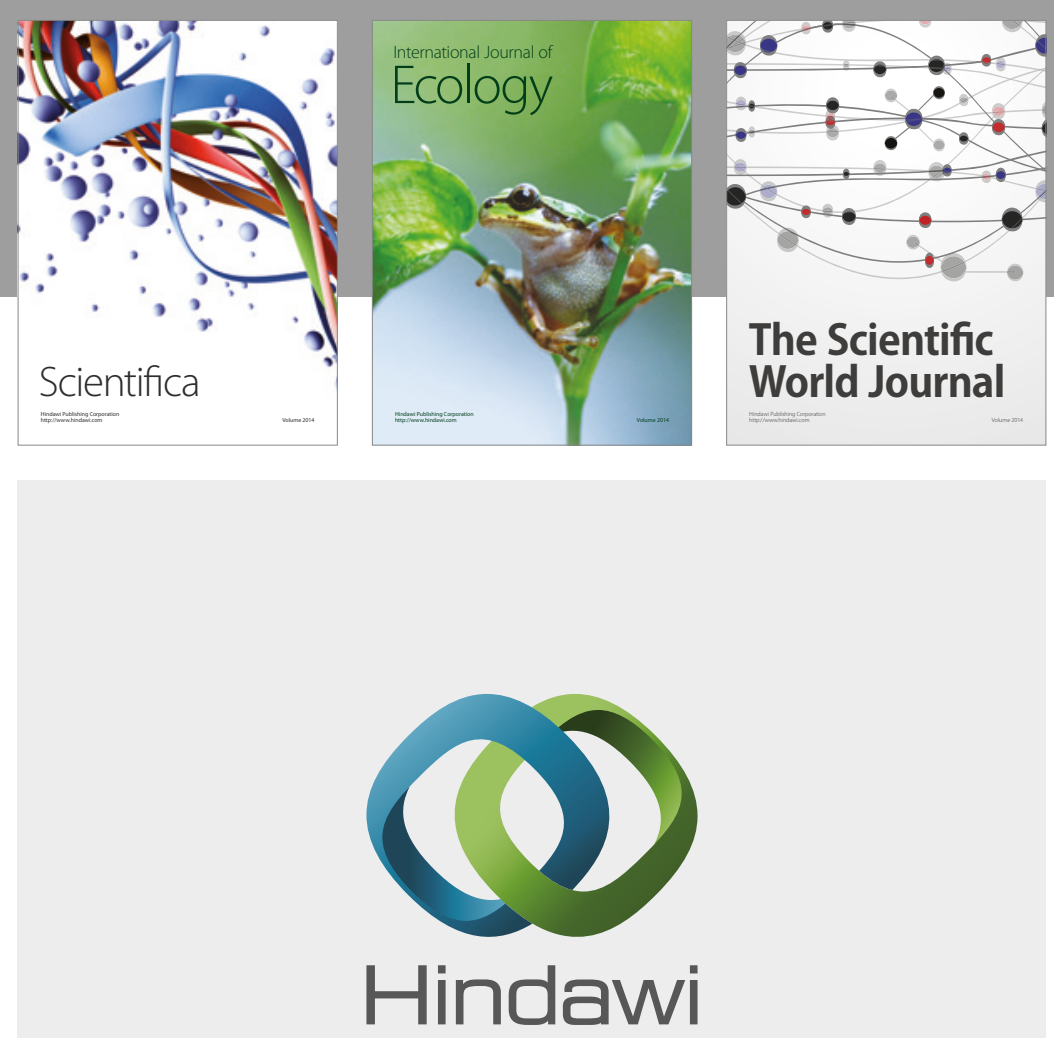

Submit your manuscripts at

https://www.hindawi.com
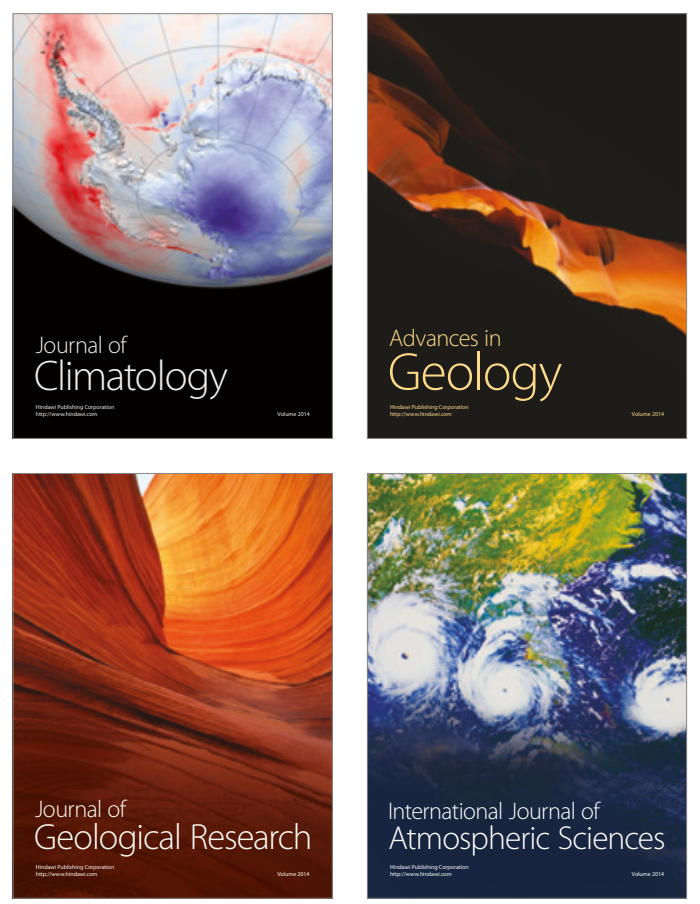

The Scientific

World Journal
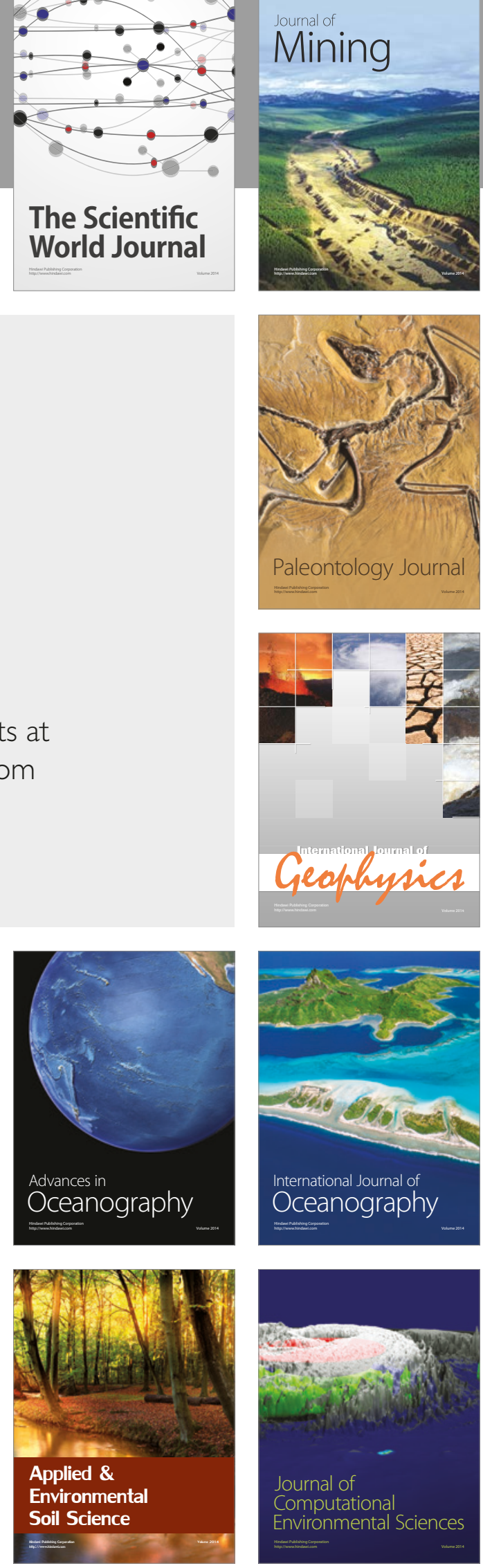